\title{
Soft inheritance: Challenging the Modern Synthesis
}

\author{
Eva Jablonka ${ }^{1}$ and Marion J. Lamb ${ }^{2}$ \\ ${ }^{1}$ The Cohn Institute for the History and Philosophy of Science and Ideas, Tel-Aviv University, \\ Tel-Aviv, Israel. \\ ${ }^{2} 11$ Fernwood, Clarence Road, London, United Kingdom.
}

\begin{abstract}
This paper presents some of the recent challenges to the Modern Synthesis of evolutionary theory, which has dominated evolutionary thinking for the last sixty years. The focus of the paper is the challenge of soft inheritance - the idea that variations that arise during development can be inherited. There is ample evidence showing that phenotypic variations that are independent of variations in DNA sequence, and targeted DNA changes that are guided by epigenetic control systems, are important sources of hereditary variation, and hence can contribute to evolutionary changes. Furthermore, under certain conditions, the mechanisms underlying epigenetic inheritance can also lead to saltational changes that reorganize the epigenome. These discoveries are clearly incompatible with the tenets of the Modern Synthesis, which denied any significant role for Lamarckian and saltational processes. In view of the data that support soft inheritance, as well as other challenges to the Modern Synthesis, it is concluded that that synthesis no longer offers a satisfactory theoretical framework for evolutionary biology.
\end{abstract}

Key words: epigenetic inheritance, hereditary variation, Lamarckism, macroevolution, microevolution.

Received: March 18, 2008; Accepted: March 19, 2008.

\section{Introduction}

There are winds of change in evolutionary biology, and they are blowing from many directions: from developmental biology (particularly the molecular aspects), from microbial biology (especially studies of mutational mechanisms and horizontal gene transfer), from ecology (in particular ideas about niche construction and studies of extensive symbiosis), from behavior (where the transmission of information through social learning is a major focus), and from cultural studies (where the relation between cultural evolution and genetic evolution is under scrutiny). Many biologists feel that the foundations of the evolutionary paradigm that was constructed during the 1930s and 1940s (Mayr, 1982) and has dominated Western views of evolution for the last 60 years are crumbling, and that the construction of a new evolutionary paradigm is underway.

In this paper we focus on one of the important challenges to the Modern Evolutionary Synthesis (referred to here as the Modern Synthesis, or the Synthesis), namely, the Lamarckian challenge, the challenge of soft inheritance. Before we do so, however, it is necessary to summarize some of the assumptions of the late $20^{\text {th }}$ century version of the Modern Synthesis, especially those that concern heredity (Jablonka and Lamb, 2005, in press):

Send correspondence to Eva Jablonka. The Cohn Institute for the History and Philosophy of Science and Ideas, Tel-Aviv University, 69978 Tel-Aviv, Israel. E-mail: jablonka@ post.tau.ac.il.
1. Heredity occurs through the transmission of germline genes. Genes are discrete units that consist of DNA and are located on chromosomes.

2. Hereditary variation is equated with variation in DNA base sequence. Cases in which acquired variations appear to be inherited can all be explained in terms of variations in DNA.

3. Hereditary variation is the consequence of (i) the many random combinations of pre-existing alleles that are generated by the sexual processes; and (ii) new variations (mutations) that are the result of accidental changes in DNA. Hereditary variation is not affected by the developmental history of the individual. There is no "soft inheritance" (in which heritable variations are the result of environmental effects, use and disuse, and other factors).

4. Selection occurs among individuals that are, at all times, well-defined entities. The target of selection is almost always the individual, which may co-evolve with its symbionts and parasites. Although some role for group selection has been acknowledged, this form of selection is assumed to be of marginal significance in evolution. The community is rarely considered as a target of selection.

5. Heritable variations have small effects, and evolution is typically gradual. Through the selection of individuals with phenotypes that make them slightly more adapted to their environment than other individuals are, some alleles become more numerous in the population. Mutation 
pressure is not an important factor in evolution. With a few exceptions, macroevolution is continuous with microevolution, and does not require any extra molecular mechanisms beyond those operating during microevolution.

6. Evolution occurs through modifications from $a$ common ancestor, and is based on vertical descent. Horizontal gene transfer has minor significance - it does not alter the basic branching structure of phylogenetic divergence. The main pattern of evolutionary divergence is therefore tree-like, not web-like.

This accepted view is now being challenged. Biologists are arguing that:

1. Heredity involves more than DNA. There are heritable variations that are independent of variations in DNA sequence, and they have a degree of autonomy from DNA variations. These non-DNA variations can form an additional substrate for evolutionary change and guide genetic evolution.

2. Soft inheritance, the inheritance of developmentally induced and regulated variations, exists and it is important. Soft inheritance includes both non-DNA variations and developmentally induced variations in DNA sequence.

3. Since many organisms (including humans) contain symbionts and parasites that are transferred from one generation of the "host" to the next, it may be necessary to consider such communities as targets of selection.

4. Saltational changes leading to evolution beyond the species level are common, and the mechanisms underlying them are beginning to be understood. Macroevolution may be the result of specific, stress-induced mechanisms that lead to a re-patterning of the genome - to systemic mutations.

5. The Tree Of Life pattern of divergence, which was supposed to be universal, fails to explain all the sources of similarities and differences between taxa. Sharing whole genomes (through hybridization, symbiosis and parasitism) and partial exchange of genomes (through various types of horizontal gene transfer) lead to web-like patterns of relations. These web-like patterns are particularly evident in some taxa (e.g. plants, bacteria) and for some periods of evolution (e.g. the initial stages following genome sharing or exchange).

In this paper we focus on the first two challenges (see also Jablonka and Lamb, 1995, 2005, 2007a, 2007b). However, the challenge of soft, epigenetic inheritance has implications that also bear on some of the other challenges to the Modern Synthesis.

\section{Inheritance through Epigenetic, Behavioral and Symbolic Variations}

The non-DNA sources of heritable variations are very often, though not invariably, developmentally induced and regulated. In Evolution in Four Dimensions (Jablonka and Lamb, 2005) we described three large categories of non-
DNA variations: epigenetic, behavioral and symbolic. However, this categorization is not without problems, because some of the categories overlap, and some are so intertwined that the distinctions become problematic (Jablonka and Lamb, 2007a). Nevertheless, these gross distinctions carve heredity at some interesting joints, so here we follow, with some qualifications, these three non-DNA dimensions, and give examples of heritable variations of each type.

\section{The soft inheritance of epigenetic variations}

Epigenetic inheritance occurs when environmentally-induced and developmentally-regulated variations, or variations that are the result of developmental noise, are transmitted to subsequent generations of cells or organisms (Jablonka and Lamb, 2005). The term "epigenetic inheritance" has been used in two overlapping ways, however (Jablonka and Lamb, 2007a). The first, which we refer to as epigenetic inheritance in the broad sense, is the inheritance of any developmental variations that do not stem from differences in DNA sequence or persistent inducing signals in the present environment. This includes cellular inheritance through the germline, and soma-to-soma informationtransfer that bypasses the germline: for example, soma-tosoma transmission through developmental interactions between mother and embryo (e.g., Clark et al., 1993). The second is cellular epigenetic inheritance, which is the transmission from mother cell to daughter cell of variations that are not the result of DNA differences. It occurs during cell division in prokaryotes and mitotic cell division in the soma of eukaryotes. Sometimes it also occurs during the meiotic divisions in the germline that give rise to sperm or eggs, so offspring may inherit epigenetic variations through the germline. The mechanisms that lead to cellular epigenetic inheritance can also lead to cell memory - to the persistence of functional and structural cellular states in non-dividing cells, such as most neurons. For example, it seems that early maternal behavior in rats has long-term effects that are associated with chromatin marks (see later) in a key gene in their offspring's brain cells (Weaver et al., 2004); changes in marks are also known to be associated with fear conditioning in rats (Miller and Sweatt, 2007).

We have called the mechanisms that underlie cellular epigenetic inheritance epigenetic inheritance systems (EISS), and distinguished between four types of EISs (Jablonka and Lamb, 2005, 2007a):

(i) Self-sustaining feedback loops. When gene products act as regulators that directly or indirectly maintain the genes own transcriptional activity, the transmission of these products during cell division results in the same states of gene activity being reconstructed in daughter cells. Such positive feedback may lead to alternative and heritable cell phenotypes, and is commonly found in fungi (Malagnac and Silar, 2003) as well as in bacteria and probably other microorganisms (Smits et al., 2006). It also plays an impor- 
tant role in the development of multicellular organisms (Ferrell, 2002).

(ii) Structural inheritance. Existing cellular structures act as templates for the production of similar structures, which then become components of daughter cells. This type of templating covers a wide spectrum of mechanisms, including prion-based inheritance in fungi (Wickner et al., 2004; Shorter and Lindquist, 2005), the inheritance of cortical structures in ciliates (Grimes and Aufderheide, 1991), and the reconstruction of what Cavalier-Smith (2004) calls "genetic membranes".

(iii) Chromatin marking. Chromatin marks are proteins and small chemical groups that are attached to DNA and influence gene activity. Relicts of these marks segregate with the DNA strands after replication, and nucleate the reconstruction of similar marks in daughter cells (Henikoff and Smith, 2007). Chromatin marks include modifiable histone and non-histone proteins that are non-covalently bound to DNA, as well as methyl groups that are covalently bound directly to the DNA.

(iv) RNA-mediated inheritance. Transcriptional states are actively maintained through interactions between small, transmissible, RNA molecules and the mRNAs or the DNA/chromatin regions with which they pair (Bernstein and Allis, 2005; Matzke and Birchler, 2005). Such interactions can be transmitted between cell and organism generations through an RNA-replication system, and/or via the interaction of the small RNAs with chromatin, which leads to heritable modifications of chromatin marks (through DNA methylation or histone modifications). RNA-DNA and RNA-RNA pairing interactions can lead not only to silencing, but also to targeted gene deletions and gene amplifications (Mochizuki and Gorovsky, 2004).

Epigenetic control mechanisms, and especially the inter-related chromatin and RNA-based EISs, are very hot topics in basic and applied research, as can be seen from the publication in 2007 of special issues of journals such as $\mathrm{Na}$ ture (vol. 447, issue 7143), Nature Review Genetics (vol. 8, issue 4), and Cell (vol. 128, issue 4), all of which were focused on epigenetics; the same year also saw the publication of the first textbook of epigenetics (Allis et al., 2007). The importance of cellular epigenetic inheritance is now beyond doubt. It seems to be ubiquitous, and over a hundred cases of inherited epigenetic variations in bacteria, protists, fungi, plants and animals have been collated by Jablonka and Raz (in press). These probably represent the tip of a very large iceberg. Here we give only a few examples of such inheritance in plants and mammals, to illustrate the scope and range of epigenetic inheritance.

A famous case of epigenetic inheritance concerns a variant of Linaria vulgaris that was described over 250 years ago by Carl Linnaeus. This variant has a floral structure that is very different from that of the normal toadflax, and Linnaeus named the new variant 'Peloria', the Greek word for 'monster'. Enrico Coen and his colleagues investigated the molecular basis of Peloria by looking at $L c y c$, the homologue of a gene that in other plants is known to control dorso-ventral asymmetry and, when mutated, leads to a similar morphological phenotype (Cubas et al., 1999). They found that the DNA sequences of the normal and peloric forms of Linaria were the same, but the pattern of DNA methylation differed: in the peloric variant the gene was heavily methylated and transcriptionally silent. The peloric form of Linaria is the result of an epimutation, not a mutation. Peloric strains are not totally stable, and occasionally branches with partially or even fully wild-type flowers develop on a peloric plant, but the epigenetic marks are transmitted to progeny for at least two generations (J. Parker, personal communication). The origin of the peloric form studied by Linnaeus is, of course, unknown, but many of the epimutations found in plants have appeared under conditions of genomic or chemical stress (Jablonka and Lamb, in press).

Animals, too, provide good examples of epigenetic inheritance. For example, Vastenhouw and colleagues found that feeding Caenorhabditis elegans with bacteria expressing double-stranded RNA that targets specific nematode genes led to morphological and physiological variations that were transmitted for at least 10 generations (Vastenhouw et al. 2006; N. Vastenhouw, personal communication). Epimutations have also been studied in Drosophila. Sollars and his colleagues investigated the effects of geldanamycin, a drug that inhibits the activity of the heat shock protein Hsp90, on the phenotype of an isogenic strain of D. melanogaster that carried a mutant allele of the Krüppel gene, which affects eye morphology (Sollars et al., 2003). They found that adding geldanamycin to the flies' food enhanced the development of the abnormal eye phenotype. Addition of the drug to the food for just one generation, followed by six generations of selective breeding, increased the proportion of flies showing the anomaly from just over $1 \%$ to more than $60 \%$. Since the strains used were isogenic, the selectable variation probably stemmed from new heritable epiallelic differences, rather than from differences in gene sequences.

Most examples of epigenetic inheritance in mammals come from studies of mice and rats. For example, Anway and his colleagues (Anway et al., 2005, 2006a, 2006b) injected pregnant females with vinclozolin (an anti-androgenic endocrine disruptor) during a sensitive period 8-15 days post coitum, and showed that the consequent abnormalities in male offspring were inherited for at least four generations. They found 15 different DNA sequences with altered methylation patterns in the $\mathrm{F}_{1}$ males that were transmitted from the $F_{1}$ to the $F_{3}$ generation.

These examples are a small sample from the many reported cases of cellular epigenetic inheritance between generations of individuals. Data reviewed by Jablonka and Raz (in press) suggest that epigenetic inheritance has been 
found in every taxon in which it has been sought, and that it can affect every type of locus in the genome (although some regions are more prone to heritable epigenetic modifications than others). The conditions inducing cellular epigenetic variations and the stability of their inheritance depend on the type of EIS and the type of organism. In many cases, the generation and transmission of epigenetic variations is responsive to external conditions and is developmentally controlled. In other words, it is "soft" inheritance.

As noted earlier, soma-to-soma transmission is also included under the large umbrella term of "epigenetic inheritance". Soma-to-soma transmission means that the germline is bypassed, but because ancestral conditions are reconstructed in the offspring, they develop phenotypes similar to those of their parents. The transmission of epigenetic information by soma-to-soma routes has been recognized in many different species of animals, and also in plants (Mousseau and Fox, 1998).

Soma-to-soma routes of transmission include those involved in transmitting or acquiring symbionts and parasites (e.g. through the ingestion of feces); transmitting products of development (e.g. chemical substances that are transmitted through the placenta and milk of mammals); and soma-dependent deposition of specific chemicals in the eggs of oviparous animals and plants. Morphological affordances or constraints (for example, maternal size) can also lead to persistent and heritable developmental effects. Transmitting ecological legacies through ecological niche construction is often part of soma-to-soma transmission (Odling-Smee et al., 2003; Turner, 2000): the ancestrally constructed environment provides the animal with a developmental resource, and through its activity it, in turn, bequeaths a similar resource to its offspring.

In animals, the behavior of the parent is frequently instrumental in the developmental reconstruction of a similar phenotype in the offspring, but the distinction between behaviorally and non-behaviorally transmitted information is often fuzzy. An excellent example of soma-to-soma transmission through maternal behavior has been studied in rats. The behavior and stress resistance of rats is influenced by the way mothers care for their young during an early sensitive period: a low level of maternal licking and grooming leads to reduced stress resistance in their offspring, whereas increased licking and grooming makes the offspring more stress resistant. The behavioral phenotype is perpetuated in the lineage, because when the daughters become mothers they reproduce the maternal-care style of their mothers (Meaney, 2001; Weaver et al., 2004).

\section{Soft inheritance in animal social evolution}

Many of the traditions found in animals are associated with observational learning, rather than more direct soma-to-soma transmission, although this is often involved as well. There are several different ways in which learning through social interactions can lead to similarity between generations and to animal traditions. They are reviewed and discussed in detail by Avital and Jablonka (2000). One well known example of how non-imitative social learning led to a new habit spreading in a population is the development of the ability of some European tits to open milk-bottles. In parts of England and elsewhere where bottles of milk were delivered to people's homes and left on their doorsteps, this behavior spread rapidly because naïve tits learnt, when in the presence of experienced individuals, that milk-bottles are a source of food. Another famous example is that of the Koshima macaques, who learnt from an innovative young female to wash sweet potatoes. A less well-known example is the tradition of opening pine-cones and eating the inner kernels, which has developed in black rats living in Jerusalem-pine forests in Israel. In this case the mothers' behavior provides conditions that enable their young to acquire this complex skill.

Learning through vocal imitation is common in song birds and marine mammals, and a few cases of motor imitation have also been reported. The imitative learning of songs in communities of birds leads to local dialects, and group-specific dialects have also been found in whales. Avital and Jablonka (2000) give many more examples of animal traditions based on non-imitative and imitative social learning (or a combination of both). The socially learnt and transmitted habits affect many aspects of an animal's life: habitat choice, food preferences and food handling, predation and defense, and all aspects of mating, parenting and social interactions with other group members. Moreover, social learning, especially early learning, can have very strong, long-term effects: some traditions are very stable, and they can evolve through cumulative additions and alterations, with one behavior being the foundation on which another is built.

\section{Symbol-based information transmission: soft inheritance in human cultural evolution}

Like the epigenetic and behavioral inheritance systems, the symbolic system enables humans to transmit information to others. We define a symbolic system as a rule-bound system in which signs refer to objects, processes and relations in the world, and also evoke and refer to other symbols within the same system (Jablonka and Lamb, 2005). The symbol-based system is human-specific, although precursors can be found in other mammals.

Human language is the most obvious example of a symbolic communication/transmission system, although mathematics, music, and the visual arts are also wonderful examples. It is not necessary to go into the many examples of human traditions that are based on symbol-based communication, because they are self-evident. Moreover, the idea that there is a process of cultural evolution based on the transmission of symbols (and variations of symbols) is generally accepted, although the complexity of this type of 
evolution is enormous. It is clear that both animal and human traditions that are based on learning cannot be captured by the gene-based Modern Synthesis model of evolution, because learning involves developmentallymediated acquisition and transmission of variations. The problem for the Modern Synthesis is that the inheritance of traditions is very soft.

\section{Soft inheritance involving DNA: the epigenome in evolution}

When considering long-term evolution, it is almost impossible to isolate one particular type of heritable variation from the others, because the different types of variations, and the processes underlying their origin and transmission, all interact. The interactions between epigenetic inheritance and the genetic system based on DNA replication is a good example of this, and sheds very interesting light on the nature of the genetic mutational processes. What has been revealed in the last few decades is that the origin of many genetic variations, especially under conditions of stress, is not random, is often predictable, and it can result in saltational changes. New genetic variation is therefore of a very different nature from that assumed in the Modern Synthesis, which proposed that evolutionarily important variations were the consequence of random mutational changes with small effects.

It has been known for many years that the rates of mutation, transposition, and recombination are lower when chromatin is condensed than when it has a more open conformation (Jablonka and Lamb, 1995, chapter 7), and that the movement of transposable elements, which is recognized as a major cause of genomic change (Kidwell and Lisch, 1997), is markedly influenced by various types of internal (genetic) and external (environmental) stress that affect DNA methylation and other components of chromatin. It is therefore clear that epigenetic variations can bias when and where in the genome genetic changes occur. However, the effect of epigenetic control mechanisms can go beyond the more or less localized mutational changes induced by local chromatin variations. Having surveyed the changes in genome structure that are part of normal development in many plants and animals, Zufall et al. (2005) suggested that developmentally regulated genome rearrangements that are under epigenetic control are an ancient feature of eukaryotes. We agree, and believe that there is a general phenomenon in which global epigenomic macrovariations, which occur during periods of stress, are inherited between generations and lead to macroevolutionary changes (Jablonka and Lamb, in press; Jablonka and Lamm, in press; Jablonka and Raz, in press). The genome-wide changes are driven by the epigenetic control mechanisms that under normal environmental conditions operate in a more limited and specific manner. Sequence studies have shown that during plant and animal phylogeny, developmental genes have been duplicated and re-used (Gu et al., 2004), and, as
Rodin et al. (2005) have suggested, epigenetic silencing may play a role in this. Epigenetic control mechanisms also play a significant role in speciation through polyploidization and hybridization, which are of central importance in plant evolution (Rapp and Wendel, 2005). Following autoand allo-polyploidization, DNA methylation patterns are dramatically altered, and genes in some of the duplicated chromosomes are heritably silenced. There is therefore a burst of new variation, which becomes the raw material for natural selection, very much as McClintock suggested many years ago (McClintock, 1984; Jorgensen, 2004; Fontdevila, 2005).

Although we do not yet know how epigenetic control systems are involved in the generation of systemic mutations, it is plausible that processes such as those seen in cilates, where epigenetic control systems bring about deletions and amplifications of genes in the developing macronucleus, also operate in other organisms under conditions of genomic and ecological stress. In general, mechanisms which are based on DNA-DNA, DNA-RNA and RNARNA pairing interactions that involve enzymatic alterations of chromatin and result in changes in DNA may underlie the systemic changes seen in stress conditions. These mechanisms are responsive to external and internal conditions, and the genetic variations that they generate, and that become subject to natural selection, are domain-specific and chromosome-specific. They are not the "random" mutations that were central to the Synthesis, nor is the evolutionary change associated with them gradual. At the time that the Synthesis was being constructed, Goldschmidt (1940) promoted the idea that systemic changes in the genome underlie macroevolution, but for many years his views were assumed to be wrong. However, recent data from many biological fronts is changing this attitude (see Shapiro, 1999; Bateman and DiMichele, 2002). It seems that systemic heritable variations, both epigenetic and genetic, do occur, and can lead to macroevolutionary changes.

\section{An extended view of heredity}

A view of heredity that incorporates non-DNA variations and the epigenetic mechanisms of their production has many other implications for evolutionary biology. Very briefly, some of these are:

1. Adaptation can occur through the selection of heritable epialleles, without any genetic change. This may be of particular importance when populations are small and lack variability, for example in situations of intense inbreeding following isolation, or following changes in reproductive strategies. The discovery of so much epigenetic variation in natural populations strengthens the view that they may play an important role in evolution (Bossdorf et al., 2008).

2. Heritable non-DNA variations may enhance the effectiveness of genetic assimilation and accommodation processes, and accelerate adaptive evolution. 
3. Heritable epigenetic variations may initiate reproductive isolation. With cellular epigenetic inheritance, differences between chromatin structures inherited from father and mother may result in hybrid offspring either failing to develop normally or being sterile, because the two sets of parental chromosomes carry incompatible chromatin marks. If there has been behavioral differentiation between populations, differences in courtship behavior, such as the preferred time or place at which it occurs, or the song dialect used, may initiate tradition-based pre-zygotic isolation.

4. Epigenetic inheritance and epigenetic control mechanisms probably played a key role in all the major transitions in evolution. For example, they were probably important during the crucial period of symbiogenesis, during the evolution of the eukaryotic chromosome, and during the evolution of language through culturally guided genetic assimilation (Jablonka and Lamb, 2006).

5. Epigenetic inheritance has constrained the evolution of ontogeny. Several developmental phenomena, such as the difficulty of reversing determined and differentiated cell states, the early segregation and quiescent state of the germline found in many animal groups, and the massive changes in chromatin structure that occur during meiosis and gamete production, may be the result of selection against transmitting to offspring the epigenetic "memories" associated with the parents' development and also chance epimutations, all of which would prevent a zygote from starting its development from a totipotent epigenetic state. In some cases, as in the evolution of genomic imprinting, selection may have favored the enhancement of germline transmitted epigenetic memories (Jablonka and Lamb, 1995).

6. The evolutionary origin of mechanisms of epigenetic and behavioral transmission, and the evolution of different strategies of transmitting information, are neglected areas of evolutionary biology, which need much more attention.

The view of heredity that is now emerging is also challenging the tree metaphor, which is based on the assumption that the pattern of evolution is a branching one, with each branch starting from a single common ancestor. If genetic exchanges, such as hybridization and infection, are common in evolution, the tree metaphor is inappropriate. There are strong arguments in favor of the conjecture that in early evolution horizontal gene transfer may have been the rule rather than the exception, and that it may still be of major importance today, especially in the evolution of microorganisms and plants (Arnold, 2006; Goldenfeld and Woese, 2007). The idea that the target of selection may be the community of interacting and transmissible genomes rather than a classical individual may also alter our understanding of evolutionary dynamics (Rosenberg et al., 2007).

\section{Conclusions}

Contrary to the established view, soft inheritance is common. Variations acquired during an individual's lifetime can be passed on through epigenetic, behavioral and symbolic inheritance. They can affect the rate and direction of evolution by introducing additional foci for selection, by revealing cryptic genetic variation, and by enhancing the generation of local genetic variations. Moreover, under conditions of stress, epigenetic control mechanisms affect genomic re-patterning, which can lead to saltational changes.

Evolutionary biology today has to incorporate soft inheritance, saltational changes due to systemic mutations, and various types of genetic exchange and cooperation. These all challenge the assumptions of the Modern Synthesis. We believe that rather than trying to continue to work within the framework of a Synthesis that was made in the middle of the last century, we now need a new type of evolutionary theory, one that acknowledges Darwinian, Lamarckian and saltational processes.

\section{Acknowledgments}

This paper is based on a lecture given by EJ in the First Evolution Workshop in Porto Alegre, Brazil, 2007. She thanks the participants for their many inputs and discussions, and the organizers who provided a stimulating social and intellectual atmosphere.

\section{References}

Allis CD, Jenuwein T, Reinberg D and Caparros M-L (2007) Epigenetics. Cold Spring Harbor Laboratory Press, Cold Spring Harbor, 512 pp.

Anway MD, Cupp AS, Uzumcu M and Skinner MK (2005) Epigenetic transgenerational actions of endocrine disruptors and mate fertility. Science 308:1466-1469.

Anway MD, Leathers C and Skinner MK (2006a) Endocrine disruptor vinclozolin induced epigenetic transgenerational adult-onset disease. Endocrinology 147:5515-5523.

Anway MD, Memon MA, Uzumcu M and Skinner MK (2006b) Transgenerational effect of the endocrine disruptor vinclozolin on male spermatogenesis. J Androl 27:868-879.

Arnold ML (2006) Evolution Through Genetic Exchange. Oxford University Press, Oxford, 272 pp.

Avital E and Jablonka E (2000) Animal Traditions: Behavioural Inheritance in Evolution. Cambridge University Press, Cambridge, $272 \mathrm{pp}$.

Bateman RM and DiMichele WA (2002) Generating and filtering major phenotypic novelties: NeoGoldschmidtian saltation revisited. In: Cronk QCB, Bateman RM and Hawkins JA (eds) Developmental Genetics and Plant Evolution. Taylor and Francis, London, pp 109-159.

Bernstein E and Allis CD (2005) RNA meets chromatin. Genes Dev 19:1635-1655.

Bossdorf O, Richards CL and Pigliucci M (2008) Epigenetics for ecologists. Ecol Lett 11:106-115. 
Cavalier-Smith T (2004) The membranome and membrane heredity in development and evolution. In: Hirt RP and Horner DS (eds) Organelles, Genomes and Eukaryote Phylogeny. CRC Press, Boca Raton, pp 335-351.

Clark MM, Karpiuk P and Galef BG (1993) Hormonally mediated inheritance of acquired characteristics in Mongolian gerbils. Nature 364:712.

Cubas P, Vincent C and Coen E (1999) An epigenetic mutation responsible for natural variation in floral symmetry. Nature 401:157-161.

Ferrell JE (2002) Self-perpetuating states in signal transduction: Positive feedback, double-negative feedback and bistability. Curr Opin Cell Biol 14:140-148.

Fontdevila A (2005) Hybrid genome evolution by transposition. Cytogenet Genome Res 110:49-55.

Goldenfeld N and Woese C (2007) Biology's next revolution. Nature 445:369.

Goldschmidt RB (1940) The Material Basis of Evolution. Yale University Press, New Haven, 438 pp.

Grimes GW and Aufderheide KJ (1991) Cellular aspects of pattern formation: The problem of assembly. Monogr Dev Biol 22:1-94.

Gu Z, Rifkin SA, White KP and Li W-H (2004) Duplicate genes increase gene expression diversity within and between species. Nat Genet 36:577-579.

Henikoff S and Smith M (2007) Histone variants and epigenetics. In: Allis DC, Jenuwein T, Reinberg D and Caparros M-L (eds) Epigenetics. Cold Spring Harbor Laboratory Press, Cold Spring Harbor, pp 249-264.

Jablonka E and Lamb MJ (1995) Epigenetic Inheritance and Evolution: The Lamarckian Dimension. Oxford University Press, Oxford, 360 pp.

Jablonka E and Lamb MJ (2005) Evolution in Four Dimensions: Genetic, Epigenetic, Behavioral, and Symbolic Variation in the History of Life. MIT Press, Cambridge, 474 pp.

Jablonka E and Lamb MJ (2006) The evolution of information in the major transitions. J Theor Biol 239:236-246.

Jablonka E and Lamb MJ (2007a) Précis of Evolution in Four Dimensions. Behav Brain Sci 30:353-365.

Jablonka E and Lamb MJ (2007b) Bridging the gap: The developmental aspects of evolution. Behav Brain Sci 30:378-389.

Jablonka E and Lamb MJ (in press) The epigenome in evolution: Beyond the Modern Synthesis. Proceedings of the Novosibirsk conference, Herald of Vavilov's Genetic Society.

Jablonka E and Lamm E (in press) The nurture of nature: Hereditary plasticity in evolution. Philos Psychol.

Jablonka and Raz (in press) Transgenerational epigenetic inheritance: Prevalence, mechanisms, and implications for the study of heredity. Q Rev Biol.

Jorgensen RA (2004) Restructuring the genome in response to adaptive challenge: McClintock's bold conjecture revisited. Cold Spring Harb Symp Quant Biol 69:349-354.

Kidwell MG and Lisch D (1997) Transposable elements as sources of variation in animals and plants. Proc Natl Acad Sci USA 94:7704-7711.

Mayr E (1982) The Growth of Biological Thought. Belknap Press, Cambridge, 974 pp.
McClintock B (1984) The significance of responses of the genome to challenge. Science 226:792-801.

Malagnac F and Silar P (2003) Non-Mendelian determinants of morphology in fungi. Curr Opin Microbiol 6:641-645.

Matzke MA and Birchler JA (2005) RNAi-mediated pathways in the nucleus. Nat Rev Genet 6:24-35.

Meaney MJ (2001) Maternal care, gene expression, and the transmission of individual differences in stress reactivity across generations. Annu Rev Neurosci 24:1161-1192.

Miller CA and Sweatt JD (2007) Covalent modification of DNA regulates memory formation. Neuron 53:857-869.

Mochizuki K and Gorovsky MA (2004) Small RNAs in genome rearrangement in Tetrahymena. Curr Opin Genet Dev 14:181-187.

Mousseau TA and Fox CW (1998) Maternal Effects as Adaptations. Oxford University Press, New York, 400 pp.

Odling-Smee FJ, Laland KN and Feldman MW (2003) Niche Construction: The Neglected Process of Evolution. Princeton University Press, Princeton, 468 pp.

Rapp RA and Wendel JF. (2005) Epigenetics and plant evolution. New Phytol 168:81-91.

Rodin SN, Parkhomchuk DV and Riggs AD (2005) Epigenetic changes and repositioning determine the evolutionary fate of duplicated genes. Biochemistry 70:559-567.

Rosenberg E, Koren O, Reshef L, Efrony R and Zilber-Rosenberg I (2007) The role of microorganisms in coral health, disease and evolution. Nat Rev Microbiol 5:355-362.

Shapiro JA (1999) Genome system architecture and natural genetic engineering in evolution. Ann NY Acad Sci 870:2335 .

Shorter J and Lindquist S (2005) Prions as adaptive conduits of memory and inheritance. Nat Rev Genet 6:435-450.

Smits WK, Kuipers OP and Veening JW (2006) Phenotypic variation in bacteria: The role of feedback regulation. Nat Rev Microbiol 4:259-271.

Sollars V, Lu X, Xiao L, Wang X, Garfinkel MD and Ruden DM (2003) Evidence for an epigenetic mechanism by which Hsp90 acts as a capacitor for morphological evolution. Nat Genet 33:70-74.

Turner JS (2000) The Extended Organism: The Physiology of Animal-Built Structures. Harvard University, Cambridge, 235 pp.

Vastenhouw NL, Brunschwig K, Okihara KL, Muller F, Tijsterman M and Plasterk RHA (2006) Long-term gene silencing by RNAi. Nature $442: 882$.

Weaver ICG, Cervoni N, Champagne FA, D'Alessio AC, Sharma S, Seckl JR, Dymov S, Szyf M and Meaney MJ (2004) Epigenetic programming by maternal behavior. Nat Neurosci 7:847-854.

Wickner RB, Edskes HK, Ross ED, Pierce MM, Baxa U, Brachmann A and Shewmaker F (2004) Prion genetics: New rules for a new kind of gene. Annu Rev Genet 38:681-707.

Zufall RA, Robinson T and Katz LA (2005) Evolution of developmentally regulated genome rearrangements in eukaryotes. $\mathrm{J}$ Exp Zool B Mol Dev Evol 304:448-455.

Editor: Angela M. Vianna-Morgante

License information: This is an open-access article distributed under the terms of the Creative Commons Attribution License, which permits unrestricted use, distribution, and reproduction in any medium, provided the original work is properly cited. 